training. One important question posed after the scenario was 'Were you concerned about Tom's relationship with Chris?' This clearly cued those who hadn't picked up on this previously. All participants including these 14 GPs made comments that they had some concerns about what was going on (see Table). One participant's comments sum it up well. 'He swiped at him at the beginning of the consultation, made a disparaging comment and did not let him go to the toilet'.

Discussion The GPs responses to this virtual reality scenario were very interesting. Most of their comments can be applied to real life scenarios but this has to take advantages over using role play often considered as the 'gold-standard'. Young children cannot be used as actors as they are unable to divorce play from reality. This becomes even more pertinent if the subject matter is child protection where there is something unpalatable about using young children who are being abused. By directly filling the questionnaires after undertaking the scenario those who had missed that this was a safeguarding scenario could be cued in a way to reconsider what had happened in a private and constructive way.

\section{G163 INVESTIGATION OF THE PROCESS AND EXPERIENCE OF GAINING MENTAL HEALTH TRAINING AS A COMMUNITY PAEDIATRIC TRAINEE}

${ }^{1} \mathrm{C}$ Coleman, ${ }^{1} \mathrm{~S}$ Haden, ${ }^{2} \mathrm{E}$ Fergusson, ${ }^{3} \mathrm{E}$ Payne. ${ }^{1} \mathrm{C}$ ommunity Paediatrics, Oxford University Hospitals Foundation Trust, Oxford, UK; ${ }^{2}$ CAMHS, Oxford Health Foundation Trust, Abingdon, UK; ${ }^{3}$ Community Paediatrics, UK

\subsection{6/archdischild-2018-rcpch.159}

Introduction The $\mathrm{RCPCH}$ General and Community Child Health $(\mathrm{CCH})$ curricula list nearly 50 competencies related to child mental health. It has long been recognised that these competencies are difficult to achieve without dedicated CAMHS training. As CAMHS is not an Allied Specialty according to the $\mathrm{RCPCH}$, an Out of Programme Experience or Training (OOPE/T) must be undertaken.

Aims To explore the process and benefits of a CAMHS training post for $\mathrm{CCH}$ trainees.

Methods A focus-group discussion at a regional training meeting, followed by collaboration with a colleague undertaking a survey of $\mathrm{CCH}$ trainees nationally, explored the attainment of mental health competencies. The steps followed to undertake formal CAMHS training were: identification of a suitable OOPT post, approval from the local department, the NHS trust, the Deanery, the RCPCH and the GMC.

Results Local and national surveys highlighted the difficulties in achieving mental health competencies within standard $\mathrm{CCH}$ training as well as lack of provision of CAMHS training. The process of applying for an OOPT took 12 months and was fundamentally facilitated by supportive, professional links between the local $\mathrm{CCH}$ and CAMHS Consultants. The $\mathrm{CCH}$ CSAC was supportive, and recognised the opportunity for competencies to be met.

Conclusions Benefits for the trainee, the CAMHS team, the $\mathrm{CCH}$ team and patient care were identified. Weekly consultant supervision and regular joint assessments provided ample opportunity to complete workplace-based assessments. There were opportunities to work within all CAMHS subspecialties. Attendance at CAMHS regional weekly registrar training meetings provided further learning and networking opportunities.

A formal training post in CAMHS led to valuable clinical experience for a future consultant career in $\mathrm{CCH}$, but would also be valuable for trainees in General Paediatrics or other sub-specialties. The post in itself naturally led to a liaison role, facilitating communication between $\mathrm{CCH}$ and CAMHS teams and benefiting patient care.

Future work to streamline the process of accessing this type of post by the RCPCH and RCPsych will be of great benefit for the specialities as a whole, to the local departments and ultimately for the patients being treated in those settings.

\section{G164 INTRODUCTION OF CPIPS WITH COLLECTION OF CLINICAL AND PATIENT JOURNEY DATA; EARLY OUTCOMES FROM 'ONE-STOP' MDT CLINIC ARE REDUCTION IN OUTPATIENT TIME, SHARED TEAM DECISION MAKING AND PROBABLE REDUCTION IN TIME TO INTERVENTIONS}

'S Pal, 1J Taylor, ${ }^{2} \mathrm{~K}$ Stohr, ${ }^{1} \mathrm{~A}$ Sansome. ${ }^{1}$ Community Paediatrics, Cambridgeshire Community Services, Cambridge, UK; ${ }^{2}$ Trauma and Orthopaedic Surgery, Cambridge University Hospitals NHS Foundation Trust, UK

\subsection{6/archdischild-2018-rcpch.160}

Background Children with cerebral palsy (CP) are recognised to have long-term morbidity. CPIPS (CP Integrated Pathway Scotland) standardises management and has been shown to reduce hip dislocation and surgical intervention. Benefits of this approach are 1)appropriate and evidence-based surveillance and 2)early referral to appropriate specialists. There are improved patient outcomes following CPIPS introduction, however limited studies reporting patient journey or financial healthcare outcome measures.

Aims To report outcomes of the graded introduction of CPIPS, initially the 'one stop' MDT clinic.

Methods Retrospective review of healthcare records of all children seen in CPIPS clinic (March-October 2017); reporting clinical and patient journey outcomes.

Results 23 children have been seen in the CPIPS 'one stop' clinics; which include a community paediatrician, paediatric orthopaedic surgeon and physiotherapist. 17\% (4/23) children had significant medication changes; such as starting muscle relaxants. $65 \%(15 / 23)$ children had subsequent list for specific specialist management, with 13\% (2/15) recieving botulinum toxin injections and $87 \%(13 / 15)$ being listed for orthopaedic surgery.

These children were previously seen separately by specialists therefore we estimate that 23 extra outpatient appointments are available. Single clinic attendance is beneficial for children (reduce time away from school) and families (reduced travel/ missed work, parking expenses, inconvenience).

Clinicians reported that shared decision making during the clinic facilitated 1)team learning 2)patient-centred holistic decision making taking into account family circumstances 3)facilitated subsequent treatments as relevant teams were present with the family to plan optimum interventions and timings.

Conclusion We present initial outcome data from the CPIPS 'one stop' MDT clinics, which have demonstrated shared decision making, positive initial comments from both professionals and families; and a theoretical financial benefit with reduced clinic usage and probable shorter time to interventions. $65 \%$ of children were subsequently booked for specialist intervention, supporting the need for 'red' alerts prior to review, ensuring appropriate children are assessed.

Further studies are needed to explore the clinical and patient journey outcomes in children with CP; alongside the outcomes 
following the introduction of CPIPS. Studies are needed to report holistic outcomes, including chronic medical conditions and the mental health to continue developing a service to support chronic, complex, and life-long conditions.

\section{G165 USING VIDEO VIGNETTES AND DOCTORS' RESPONSES TO ASSESS THE EFFICACY OF PAEDIATRIC SIMULATION}

${ }^{1} \mathrm{M}$ Malley, ${ }^{2} \mathrm{M}$ Ko, ${ }^{3} \mathrm{M}$ Monaghan. ${ }^{1}$ West Middlesex Hospital, London, UK; ${ }^{2} \mathrm{FCransley}$ Hospice, Kettering, UK; ${ }^{3}$ Epsom General Hospital, London, UK

10.1136/archdischild-2018-rcpch. 161

Aims Paediatric simulation is an established part of medical student training. However there remain many ways to assess its efficacy. We aimed to assess the efficacy of a simulation programme using video-based clinical vignettes and comparing student responses to established paediatric doctors.

Method We created a paediatric simulation programme for 87 fifth year medical students. The programme involved two-hour theory session followed by a two-hour high fidelity simulation in groups of five.

Twelve clinical vignettes were created based on the videos from 'Spotting The Sick Child' online resource (with permission). The students stated how sick they thought the child was for each scenario on a scale of 1 to 10 , where 10 was 'immediate PICU admission' and 1 was 'immediate discharge'. This quiz was carried out pre- and post-course. Twenty post$\mathrm{MRCPCH}$ paediatricians carried out the same quiz. The average of their scores was considered the 'gold standard'. The deviation between the paediatrician and student scores was calculated both pre- and post-programme. Students also rated their confidence level in assessing and managing the cases before and after the programme.

Results Overall, students' responses were significantly closer to the doctors' mean post session $(\mathrm{p}=0.0048)$. This was particularly pronounced in respiratory distress, dehydration and DKA scenarios. The average value of students' confidence level for assessing paediatric emergencies pre- and post-simulation were $2.91 / 5$ and $3.58 / 5$, respectively $(\mathrm{p}<0.001)$. Similarly their reported confidence in managing the emergencies was $2.36 / 5$ pre-session and 3.36/5 post-session $(\mathrm{p}<0.000005)$.

Conclusion Video vignettes provided a novel approach to assessing the efficacy of a paediatric simulation programme. Students' responses more closely correlated with more experienced specialist physicians after the session compared to presession. The convergence of the paediatricians' and students' scores post-session was validated by the students' self-reported increased confidence level in assessing and managing the scenarios post-session. This supports the utility of this method for assessing the clinical educational efficacy of paediatric simulation programmes.

\section{G166 USEFULLNESS OF SOCIAL EMOTIONAL AND ADAPTIVE BEHAVIOUR AS PART OF TWO YEAR NEURODEVELOPMENTAL ASSESSMENTS}

${ }^{1} \mathrm{D}$ Metheniti, ' $\mathrm{M}$ Parmar, ${ }^{1} \mathrm{P}$ Southernwood, ${ }^{2} \mathrm{~S}$ Beasley, ${ }^{1} \mathrm{~N}$ Merchant. ${ }^{1}$ Paediatrics and Neonatology, West Hertfordshire NHS trust, Watford, UK; ${ }^{2}$ Peace Children's Centre Department of Physiotherapy, West Hertfordshire NHS Trust, Watford, UK
Introduction Neurodevelopmental outcome assessment provides basis for benchmarking and early intervention. Bayleys scale of infant development (BSID) is often used in 2 year assessments of high-risk infants, however there are time constraints in completing these assessments. Social, emotional and adaptive processes like self regulation and executive function which are increasingly being shown to predict later life and behaviour outcomes are often not captured in these assessments.

Aim To study if completion of social emotional and adaptive questionnaire of BSIDIII will be useful as part of the 2 year assessments.

Methods Social-emotional/adaptive parental questionnaire was given for all preterms born $<30$ weeks at the 2 year neurodevelopmental clinic. Demographic and infant data was collated along with the composite scores of the cognitive, language and motor, social emotional and adaptive scales of BSIDIII. Moderate delay was defined as composite score of 70-84 and severe delay as $<70$. Data was analysed using Microsoft Excel and STATA.

Results 19/48 (40\%) who returned the questionnaire had 2 year assessments at a mean age of 25 months. Table 1 shows the mean composite scores of different scales. There was significant correlation with general adaptive composite (GAC) and motor composite $(p=0.04,95 \% \mathrm{CI}-1.29$ to $-0.33)$. A significant correlation was also seen with language composite and social adaptive domain $(p=0.042,95 \%$ CI -0.7 and -0.15$)$. There was no correlation seen with social emotional scale and motor/language/cognitive scales.

Abstract G166 Table 1 Mean composite scores from BSID subscales (GAC-general adaptive composite, CON-conceptual adaptive domain, SO- Social adaptive domain, PR-Practical adaptive domain)

\begin{tabular}{lllllllll}
\hline & Cognitive & Language & Motor & SE & GAC & CON & SO & PR \\
\hline Mean & 95.6 & 96.6 & 86.5 & 111.5 & 98.9 & 98.8 & 103.4 & 94.9 \\
Composite & $(3.1)$ & $(6.2)$ & $(2.7)$ & $(6.1)$ & $(5.6)$ & $(5.1)$ & $(4.1)$ & $(5.7)$ \\
scores(SD) & & & & & & & & \\
\hline
\end{tabular}

Conclusion Parental questionnaire using the social-emotional and adapative behaviour items can be used as screening tool to identify high-risk infants requiring speech and physiotherapy intervention. This may allow streamlining of clinics that run the 2 year assessments. This data needs to be validated with a larger cohort.

\section{G167 PROMOTING TECHNOLOGY ENHANCED LEARNING: ASSESSING VIEWS AND EFFICIENCY OF MOBILE PHONES IN CLINICAL PRACTICE}

\begin{abstract}
${ }^{1,2} \mathrm{C}$ Junk, ${ }^{2}$ J Wallace, ${ }^{1} \mathrm{P}$ Mallett, ${ }^{1,2} \mathrm{~A}$ Thompson. ${ }^{1}$ Department of Paediatric Simulation and Education, Royal Belfast Hospital for Sick Children, Belfast, UK; ${ }^{2}$ Department of Paediatrics and Child Health, Royal Belfast Hospital for Sick Children, Belfast, UK
\end{abstract}

\subsection{6/archdischild-2018-rcpch.163}

Aims Technology-enhanced learning, specifically the use of mobile devices by Healthcare professionals has transformed many aspects of clinical practice. ${ }^{1}$

Some healthcare organisations are reluctant to advocate the staff use of mobile phones due to the risks associated with 